\title{
An Experimental Approach to the Pathogenesis of "Pipestem" Fibrosis (Symmers' Fibrosis of the Liver)
}

\author{
Zilton A Andrade ${ }^{+}$, Luciana M Silva, Marcia Maria de Souza
}

Laboratório de Patologia Experimental, Centro de Pesquisas Gonçalo Moniz -FIOCRUZ, Rua Valdemar Falcão 121, 40295-001 Salvador, BA, Brasil

Pathogenesis of schistosomal hepatic fibrosis ( "pipestem" fibrosis of the liver) was investigated by means of the murine model. Although worm load appears as the main pathogenetic factor, alone it is not sufficient to produce that characteristic lesion. By comparing the findings in animals with heavy and prolonged Schistosoma mansoni infection, which developed or not "pipestem" fibrosis, it was observed that the lesion was more frequent in intact animals than in the splenectomized one. However, the size of the spleen, the number of recovered worms, the number of eggs per gram of liver tissue, the level of serum idiotype and anti-idiotype antibodies, the size and volume of periovular granulomas formed in the liver, all that failed to show statistically significant differences between the two groups. After analysing all these data, other factors, that apparently have been hitherto negleted, rested to explain the findings. Among them, the timing and sequence of the egg-induced intrahepatic vascular changes seemed crucial. The sequential development of intrahepatic portal vein obstruction, followed by the opening of periportal collateral veins and the continous arrival of schistosome eggs going to be lodged into the latter, appeared as essential steps in the pathogenesis of "pipestem" fibrosis.

Key words: Schistosoma mansoni - "pipestem” fibrosis - pathogenesis

One of the most characteristic, if not pathognomonic, lesion of human pathology is that first described by Symmers (1904), and since then known as "pipestem" fibrosis of the liver. It represents the morphological counterpart of hepatosplenic schistosomiasis, which is clinically characterized by hepato-splenomegaly, portal hypertension (esophageal varices) and variable degrees of pancytopenia (hypersplenism), usually in the absence of signs of hepato-cellular failure. The lesion involves every branch of the portal tree, the fibrous tissue that enlarges the portal spaces appearing at the cut surface of the liver as whitish plaques against a background of normal-looking parenchyma (Fig. 1).

Lichtenberg and Sadun (1968) listed the following anatomical characteristics for the lesion: (a) grossly evident, diffuse, stellate fibrosis and enlargement of the large and medium-sized portal fields; (b) variable portal inflammatory infiltration and crowding of the portal areas with schistosome eggs and granulomas (active stage) or scar tissue containing egg shells and pigment (late stage); (c) conservation of the lobular architecture, except for porto-central fibrous banding or post-necrotic scar-

Financial support was provided by PAPES-1 (FIOCRUZ).

+Corresponding author. Fax: +55-71-356.4292

Received 16 April 1997

Accepted 30 June 1997 ring usually confined to the subcapsular parenchyma; (d) relatively normal hepatocytic structure and function; (e) destructive lesions of the intrahepatic portal radicles.

Pathogenesis of "pipestem" fibrosis is apparently simple. Heavily infected patients develop periportal fibrosis with vascular obstruction due to the deposition of numerous schistosome eggs along the trajectory of the portal vein. This explanation is in keeping with quantitative findings obtained by Cheever (1965) who counted worms by doing perfusion of the portal system of cadavers. It also agrees with the fact that severe schistosomiasis with "pipestem" fibrosis is not to be seen in subjects with mild schistosome infections. As a matter of fact, a good rule of the general pathology of helminthic infections indicates that severe clinical manifestations are related to heavy worm burden. On the other hand, it is common experience that people with heavy Schistosoma mansoni infection, sometimes passing 500 to 2,000 or more eggs per gram of feces, do not necessarily evolve toward hepatosplenic schistosomiasis (Coura \& Conceição 1981). Perhaps, even the majority of severely infected patients will escape from developing the characteristic lesion of "pipestem" liver fibrosis. Therefore, although heavy worm load appears as an essential factor for the pathogenesis of "pipestem" fibrosis, this factor alone is not sufficient. Other factor or factors may play additional, probably crucial, role. 


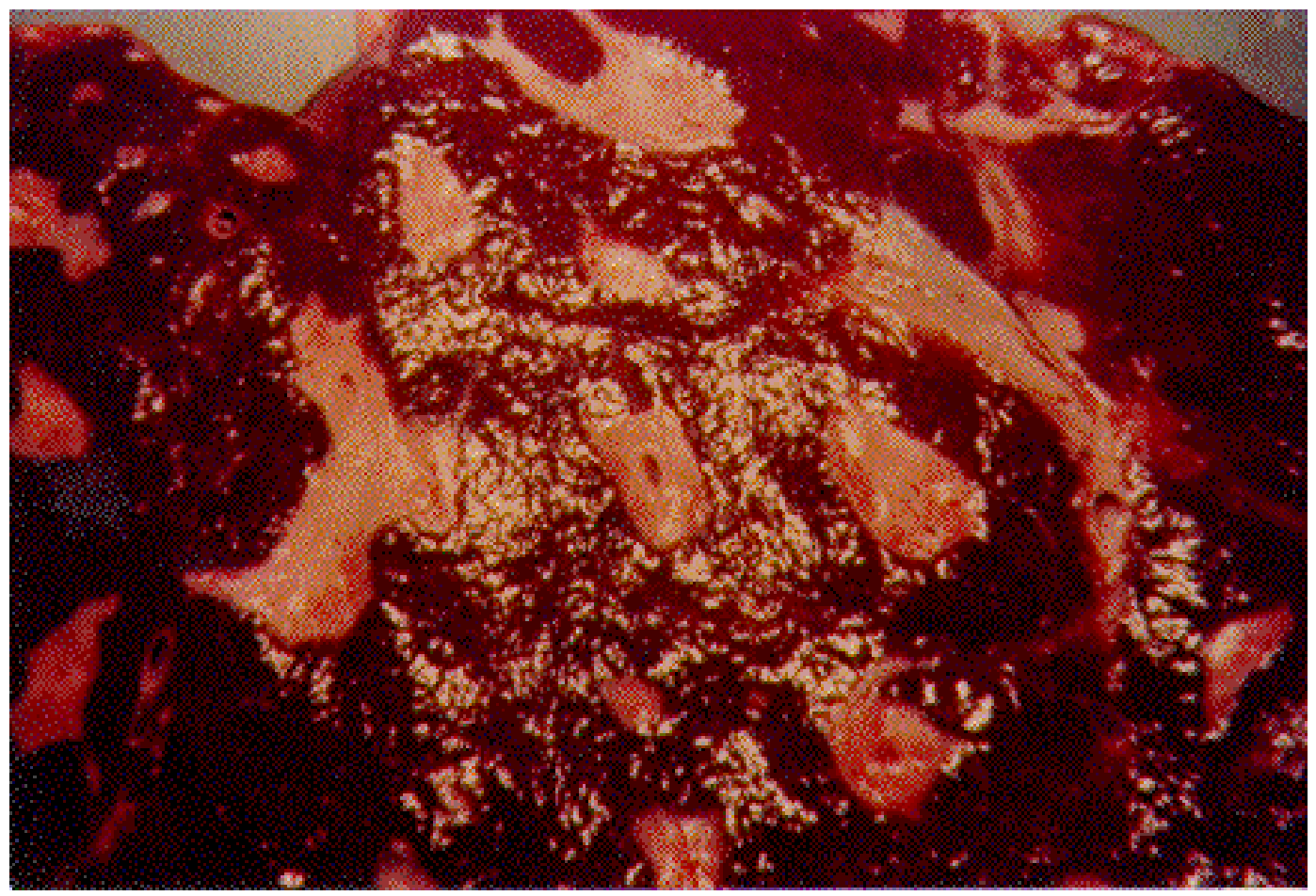

Fig. 1: cut surface of the liver from a case of hepatosplenic schistosomiasis. The whitish fibrous tissue forms plaques that enlarges the portal spaces. The liver parenchyma appears congested, but otherwise with normal aspect.

Attempts to investigate the problem of additional factors in the pathogenesis of hepatosplenic schistosomiasis in humans have not been very successful. This subject has been reviewed by Prata (1991). Considerable attention has been paid to genetic background, especially to race and HLA determinants of hepatosplenic patients, but results have so far been inconclusive. The age of the patients when first infected, the frequency of exposure to contaminated waters, the failure to generate anti-idiotypic antibodies having a modulatory influence on periovular granulomatous formation represent the main lines of research. Results have been so variable, that the need for a good experimental model is well recognized.

\section{EXPERIMENTAL APPROACH}

Warren (1966) observed that mice, infected with 1-2 worm pairs of $S$. mansoni and left for prolonged periods of time (more than 16 weeks), developed systematized periportal fibrosis due to a concentration of periovular granulomas and fibrosis along the portal spaces. Although the lesion was only observed microscopically, its similarity to human "pipestem" fibrosis was unquestionable. At first it was stated that chronic infection in mice did not lead to periportal fibrosis (Cheever 1965) and this was probably a common opinion, since this interesting model was not further explored for some time after Warren's paper.

While performing studies on S. mansoni infection in chimpanzees, Sadun et al. (1970) observed the development of typical, gross and microscipical "pipestem" fibrosis in some heavily infected animals. Difficulties of several types, especially concerning the obtaining, maintenance and management of these primates, turned the model of little practical usefulness.

In the last 10 years we became interested in the mouse model of "pipestem" fibrosis. Our attention to the subject came by sheer chance. While investigating whether fibrosis in late periovular granulomas would also be reversible after treatment, we submitted mice to light infection with only 15-20 cercariae in order to decrease mortality, and waited up to 20 weeks before treating them. As a result we observed that some mice with prolonged infection not only developed periportal fibrosis as Warren (1966) had described, but that periportal fibrosis was also susceptible to degradation following specific chemotherapy (Andrade \& Grimaud 1986). Our interest specially arouse be- 
cause of earlier findings with the technique of plastic vascular injection followed by corrosion in strong acid for the obtaining of portal vein casts. $S$. mansoni infected mice revealed the development of a periportal cuffing of collateral vessels (Fig. 2, Andrade \& Brito 1981). This finding appeared then as the missing link necessary to explain the somewhat curious distribution of eggs along periportal spaces (Andrade 1987). The questions now were: how frequently the periportal lesion developed in mice with chronic infection and how representative of human schistosomisis it was? The material then studied included a group of outbred mice and several inbred strains. This study was complemented with material taken from the files of the Laboratory of Parasitic Diseases, NIH, Bethesda, MD, USA. The development of schistosomal periportal fibrosis occurred in both outbred and inbred strains of mice, indicating that genetic background did not seem to be a crucial factor. The percentages of "pipestem" fibrosis was quite variable in the several groups of mice, in general around $50 \%$. Table shows the distribution of the lesions according to mouse strains. Mice that failed to develop the "pipestem" lesion showed scattered isolated granulomas instead. As to the significance of the model, it was considered valid as an experimental model of human "pipestem" fibrosis. Actually, the lesion was represented by a concentration or periovular granulomas along periportal areas, rather than a diffuse periportal fibrosis. But, there occurred portal vein obstructive lesions and a certain amount of extra-granulomatous periportal fibrosis (Andrade \& Cheever 1993).

A mouse model with marked and dense periportal fibrosis was described by Henderson et al. (1993) in about $20 \%$ of male CBA/J inbred mouse chronically infected with approximately $45 \mathrm{~S}$. mansoni cercariae. With this model "pipestem" fibrosis was accompanied by massive splenomegaly,

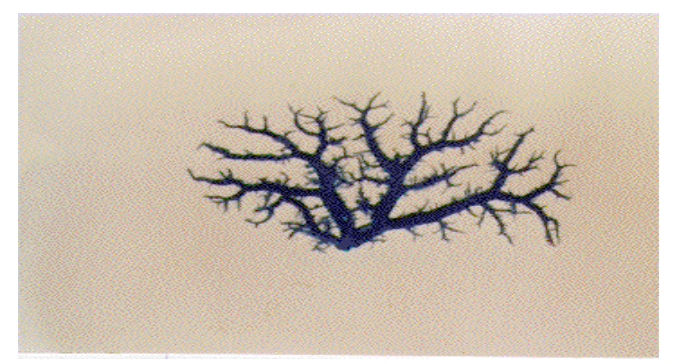

Fig. 2: vascular plastic cast from the portal system of a mouse with chronic schistosomiasis. Numerous fine collaterals give a hairy appearance to the portal vein branches.

a feature not so impressive in previous studies. Furthermore, the animals with "pipestem" fibrosis exhibited a peculiar anti-idiotypic profile against anti-egg antigen, which was distinct from those mice that did not develop the lesion. Similar serological findings have been obtained by the same group in hepatosplenic patients (Colley et al. 1986). This led to the concept that immunological modulatory failure during periovular granuloma formation is an important pathogenic factor for the development of "pipestem" fibrosis.

\section{RECENT EXPERIMENTS}

Generalities - Experiments in our laboratory have been performed in outbred albino young (15$18 \mathrm{~g}$ ) Swiss mice of both sexes. Animals were submitted to infection by the transcutaneous route with about 20-30 recently shed S. mansoni cercariae. Only those animals which were eliminating viable eggs in the stools were included in the study. Sacrifice took place about 20-22 weeks from cercarial exposure. Paraffin embedded histological sections stained with sirius red (Junqueira et al. 1979) were then examined with the low power of the micro-

TABLE

General data on the murine model of schistosomal "pipestem" fibrosis of the liver

\begin{tabular}{|c|c|c|c|c|c|}
\hline \multirow[t]{2}{*}{ Strain } & \multirow{2}{*}{$\begin{array}{c}\text { Duration of } \\
\text { infection (wks) }\end{array}$} & \multicolumn{2}{|c|}{ "Pipestem" fibrosis } & \multirow{2}{*}{$\begin{array}{r}\text { Eggs/liver } \\
(\mathrm{x} 1,000)\end{array}$} & \multirow{2}{*}{$\begin{array}{l}\text { Spleen wt } \\
\text { (mg) }\end{array}$} \\
\hline & & Yes $(\%)$ & No $(\%)$ & & \\
\hline Outbred & 23 & $27(54)$ & $23(46)$ & 30 & No data \\
\hline C57BL/8 & 20 & $6(42.8)$ & $8(57.2)$ & 24 & 249 \\
\hline C57B $1 / 6$ & 52 & $21(70)$ & $9(30)$ & 62 & 217 \\
\hline $\mathrm{BALB} / \mathrm{c}$ & 52 & $8(80)$ & $2(20)$ & 50 & 225 \\
\hline $\mathrm{BALB} / \mathrm{c}$ & 69 & $10(79.9)$ & $3(23.1)$ & 74 & 265 \\
\hline $\mathrm{C} 3 \mathrm{H} / \mathrm{HeN}$ & 22 & $6(66.6)$ & $3(33.3)$ & 25 & 336 \\
\hline $\mathrm{C} 3 \mathrm{H} / \mathrm{HeN}$ & 41 & $7(63.6)$ & $4(36.4)$ & 27 & 407 \\
\hline $\mathrm{C} 3 \mathrm{H} / \mathrm{HeN}$ & 52 & $5(100)$ & 0 & 46 & 420 \\
\hline
\end{tabular}

Abstracted from Andrade ZA \& Cheever AW 1993 Int J Exp Pathol 74: 195-202. 
scope. Infected mice, harboring 1-2 pairs of worms presented with either one of the following lesions: isolated periovular granulomas (Fig. 3) or periovular granulomas and fibrosis preferentially distributed along the periportal spaces (Fig. 4). Usually, the portal spaces in this last group appeared amplified by fibrosis and stood out as dense stellate areas on a background of unstained parenchyma, forming a very characteristic lesion (Fig. 5). Discrimination between the two types of lesions was usually clear-cut. Only rarely did a mixture of the two lesions occur, in such a way as to make the decision difficult.

Role of the spleen and other factors - With this very simple basic methodology, experiments were performed to compare "pipestem" positive and negative animals according to the following parameters: the influence of the spleen (splenectomized versus sham-operated animals), spleen weight and splenic microscopic changes, worm load (number of eggs per gram of liver tissue), morphometric measurements of periovular granulomas and general immunological abnormalities (idiotype and anti-idiotype anti-schistosome antibodies).

In these recent sets of experiments (Andrade et al. manuscript in preparation), "pipestem" fibrosis of the liver appeared in $38.3 \%$ of 94 mice. In 60 splenectomized animals $(61.8 \%)$ the lesion was present in $15(25 \%)$ and absent in 45 (75\%). Among $34(36.2 \%)$ sham-operated animals the lesion de- veloped in $21(61.8 \%)$ and was absent in 13 (36.2\%). These results pointed out, in a statistically significant way, that the presence of the spleen does favour the development of "pipestem" fibrosis. However, no clear-cut differences appeared between splenectomized and sham-operated animals regarding the levels of serum antibodies against $S$. mansoni or the levels of anti-idiotypic antibodies tested against whole adult worm antigens, the size of the spleen (measured as splenic index), and the number of eggs per gram of liver tissue or the number of worms recovered from perfusion of the portal system.

Experiments with undernourished mice - An attempt to investigate the influence of nutrition on the development of "pipestem" fibrosis was performed in two groups of mice (Coutinho et al. manuscript in preparation): one was maintained on a basically low-protein diet that has been intended to represent the basic regional diet of poor people living in endemic areas for schistosomiasis in northeastern Brazil (Coutinho 1980); the other was a control group of mice maintained on a normal commercial balanced chow. The same basic methodology described above was used. Undernourished animals reached only half the weight of the controls at the end of experiments. They had fewer worms and fewer eggs per worm pairs, smaller liver and spleen, both in absolute terms and in relation to body weight. Hepatic periovular granulomas were significantly smaller than those in con-

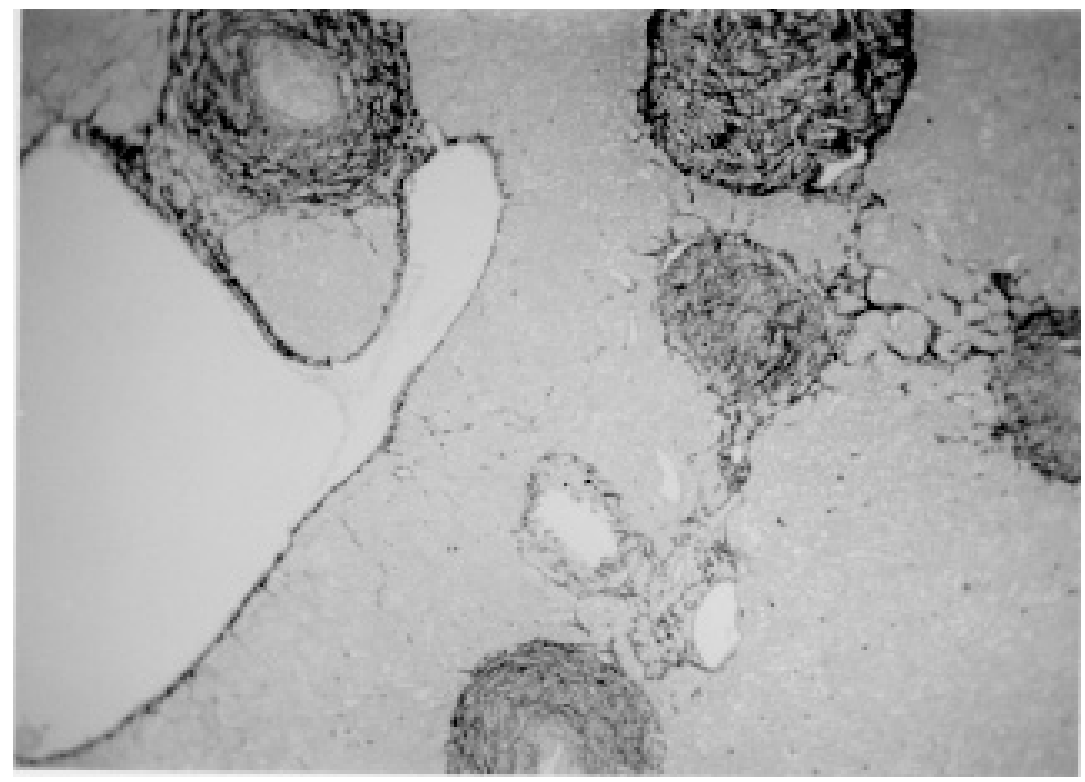

Fig. 3: isolated periovular granulomas. These granulomas are large, contains abundant collagen fibers and form discrete nodules scattered within the liver. Picro-sirius-red method for collagen, X 150. 


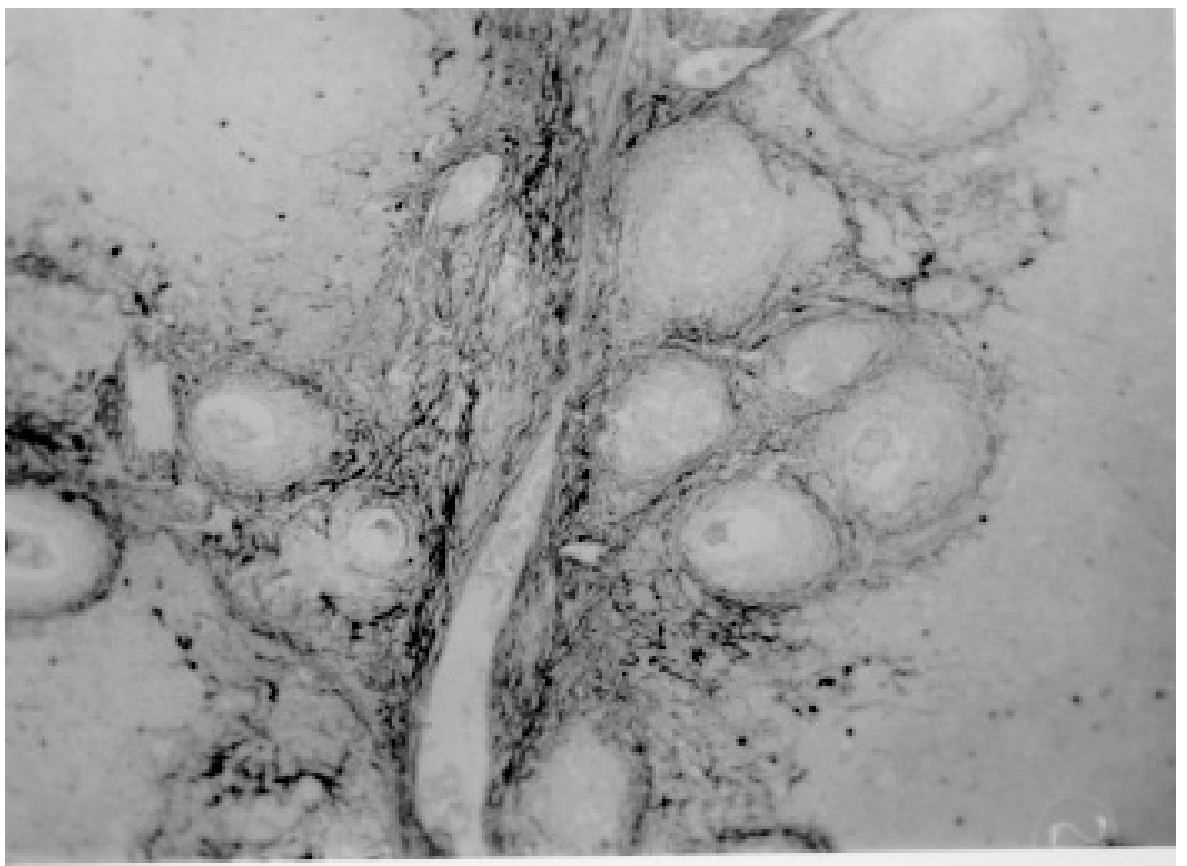

Fig. 4: periovular granulomas are numerous and show a tendency to accumulate along the periportal space. Note that the portal space is amplified by fibrosis and the main portal vein branch presents sub-intimal thickening. Picro-sirius-red method for collagen, X 150.

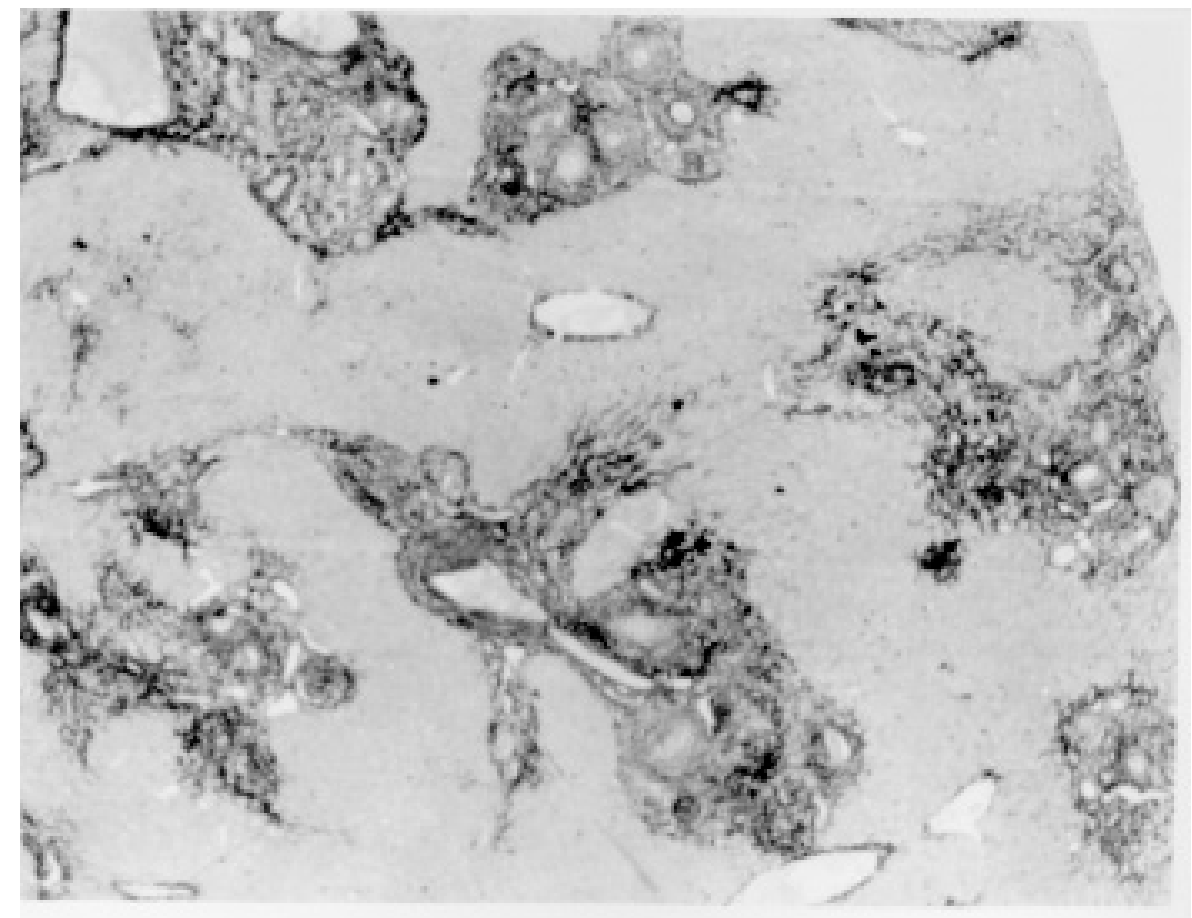

Fig. 5: portal fibrosis appears as dark plaques of fibrous tissue on a background of lightly stained normal-looking hepatic parenchyma in this preparation stained by the Picro-sirius-red method for collagen. This represents the essential feature of the murine model of schistosomal "pipestem" fibrosis of the liver. X100. 
trol mice. None of the 16 mice maintained on deficient diet developed "pipestem" fibrosis at the end of 16 weeks of infection, while 7 (43.7\%) out of 16 on a normal diet did. The total amount of fibrosis (collagen) in the liver was decreased in undernourished mice as compared to controls when evaluated both by morphometry and biochemistry (hydroxiproline).

\section{DISCUSSION}

The task of evaluating the results obtained in our laboratory with the murine model is not a simple one. First of all we have to question whether the model really represents the lesion seen in advanced schistosomiasis of man. As a matter of fact, this contention appeared even before (Cheever 1965) the model was first described by Warren (1966). A critical appraisal of the murine model of "pipestem" fibrosis has already been attempted (Andrade \& Cheever 1993). Weighing similarities and differences, it has been concluded that the model stands up as a miniature of the human "pipestem" fibrosis and, being so, the model is worth of experimental investigation. Not only the eggs and the granulomas they elicited were concentrated within the portal spaces, but there were also extra-granulomatous fibrosis and vascular lesions (Fig. 6). As in the human lesion, portal vas- cular lesions include angiogenesis, phlebosclerosis, narrowing and obstruction (Fig. 7).

Considering the worm load of the animals, one important point was raised by Cheever (1968). He estimated that the heaviest infection reported in man at autopsy have seldom been grater that 5 worm-pairs per kg of body weight, whereas in a $20 \mathrm{~g}$ mouse the lightest infection corresponds to about 50 worm pairs per kilogram of body weight. Therefore, animals in the experiments reported above were all heavily infected. They harbored 12 worm pairs and their livers contained from 6,400 to 10,300 schistosome eggs. This means that in animals developing periportal fibrosis other factor(s) might have influenced the distribution of eggs all along the periportal spaces.

The murine "pipestem" fibrosis is neither seen in early (8-12 weeks), nor in late, massive (50 cercariae up) infections. In these latter infections eggs are not orderly distributed and concentrated in portal spaces, but appear throughout the liver, producing fibrosis and distorting the hepatic vasculature without a definite pattern. The importance of the "right" number of eggs and the timing of their deposition within the liver, probably in concert with a sequence of vascular changes are therefore apparent. In view of the negative results obtained when such factors as genetical, immunological and

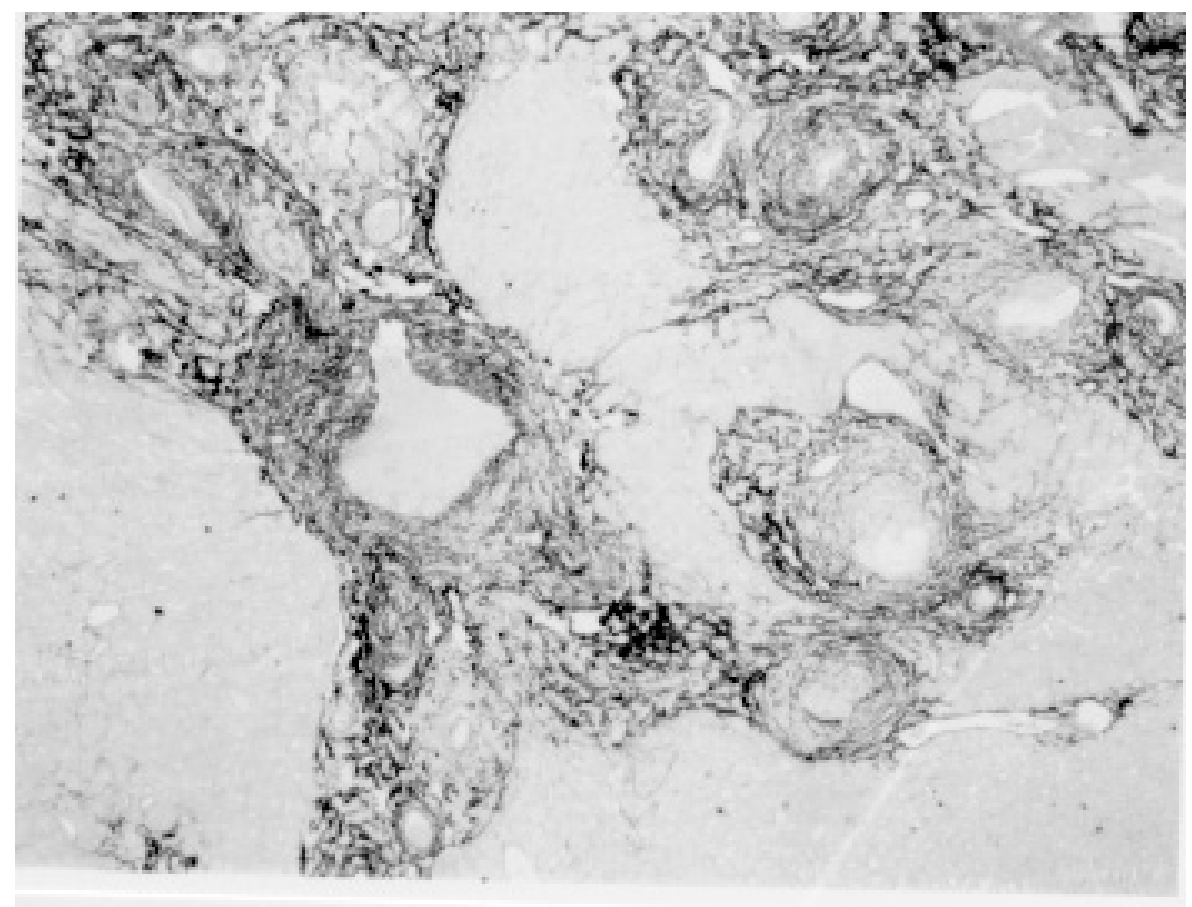

Fig. 6: murine "pipestem" fibrosis. Besides the periovular granulomas and the bands of collagen, the periportal lesion appears well vascularized, some of the vessels appearing with thickening of their walls. Picro-sirius-red method for collagen. $120 \mathrm{X}$. 


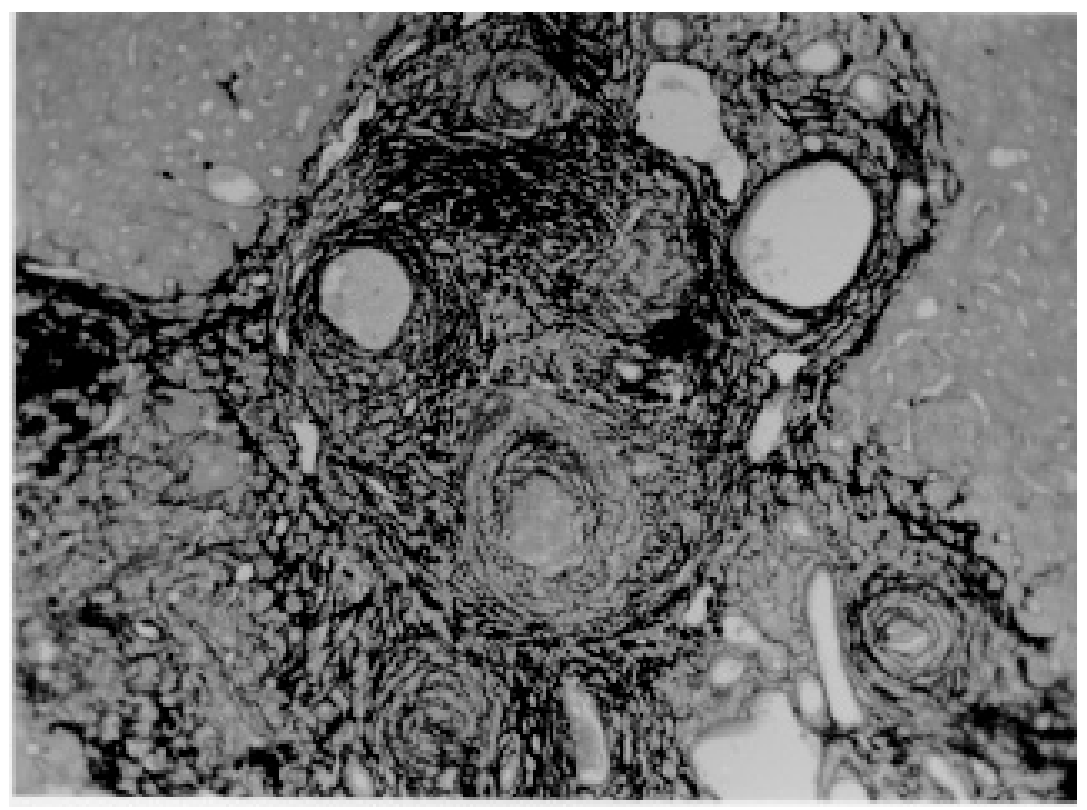

Fig. 7: portal fibrosis and periovular granulomas. The vessels are numerous, the majority are small and represented by slits, other are dilated and thickened. Picro-sirius-red method for collagen. 250X.

parasitological features were investigated, the importance of the factor sequential vascular changes assumes a prime importance for explaining the pathogenesis of "pipestem" fibrosis in mice.

Studies with injection of plastic material into the portal vein, followed by corrosion in strong acid, have yielded casts which showed numerous collateral vessels sprouting out from the branches of the intrahepatic portal vein in mice chronically infected with S. mansoni, a finding not seen in noninfected controls (Andrade \& Brito 1981). These vascular changes allow for the schistosome eggs to be lodged in small vessels distributed along the periportal tissues.

For the development of this crucial vascular alteration, it is presumably necessary a series of sequential events: a somewhat disseminated and sudden obstruction of portal radicles by periovular granulomas, generation of intrahepatic portal hypertension, opening of periportal collaterals, arrival of schistosome eggs into these new vascular canals. Factors influencing this chain of events are probably multifactorial. Of course, the production of numerous parasite eggs is essential. A proper (normal) immunological response is also important, as the data from undernourished mice indicated. But the timing of the vascular changes and the arrival of new eggs seem essential for the end-result of "pipestem" fibrosis.

Formation of peri-portal vascular cuffing was a prominent finding seen in human "pipestem" fi- brosis when plastic casts were obtained with the injection/corrosion methodology (Bogliolo 1957, Andrade \& Cheever 1971).

It seems still premature to say that the pathogenesis of "pipestem" fibrosis of the liver is the same for mouse and man.

In some patients with hepatosplenic schistosomiasis pulmonary arteritis develops as a complication. It has been shown that when a few eggs reach the lungs they are lodged in alveolar precapillaries, forming discrete granulomas. Severe lesion does not result from gradual accumulation of eggs, but from a sudden and massive embolization to the lungs. Then, intra-pulmonary hypertension causes dilatation of fine collaterals that come out at right angles from large and medium-sized pulmonary arteries. If the arrival of eggs continues, many of them are stopped at the entrance of those collaterals causing severe arterial and arteriolar damage, with vascular obstruction that results in chronic "cor pulmonale". This is a good example of vascular changes occurring in concert with parasite stimuli to produce a sequence of changes toward a characteristic schistosomal picture (Andrade \& Andrade 1970). No particular racial or immunological features were apparent in those 14 out of 78 hepatosplenic patients (17.9\%) who concomitantly developed chronic schistosomal "cor pulmonale".

The pathogenesis now postulated for the hepatic lesion is basically the same for both the he- 
patic and the pulmonary lesions of advanced schistosomiais.

\section{REFERENCES}

Andrade ZA 1987. Pathogenesis of pipe stem fibrosis of the liver (experimental observation on murine schistosomiasis). Mem Inst Oswaldo Cruz 82: 325334.

Andrade ZA, Andrade SG 1970. Pathogenesis of pulmonary schistosomiasis. Am J Trop Med Hyg 19: 305-310.

Andrade ZA, Brito PA 1981. Evolution of schistosomal hepatic vascular lesions after specific chemotherapy. Am J Trop Med Hyg 30: 1223-227.

Andrade ZA, Cheever AW 1971. Alterations of the intrahepatic vasculature in hepatosplenic schistosomiasis mansoni. Am J Trop Med Hyg 20: 425-432.

Andrade ZA, Cheever AW 1993. The characterization of the murine model of schistosomal periportal fibrosis ("pipestem" fibrosis). Int J Exp Med 74: 195202.

Andrade ZA, Grimaud J-A 1986. Evolution of the schistosomal hepatic lesions in mice after curative chemotherapy. Am J Pathol 124: 59-65.

Bogliolo L 1957. The anatomical picture of the liver in hepatosplenic schistosomiasis mansoni. Ann Trop Med Parasitol 51: 1-14.

Cheever AW 1965. A comparative study of Schistosoma mansoni infections in mice, gerbils, multimammate rats and hamsters. II - Qualitative pathological differences. Am J Trop Med Hyg 14: 227-238.

Cheever AW 1968. A quantitative post-mortem study of schistosomiasis mansoni in man. Am J Trop Med Hyg 17: 38-64.

Colley DG, Garcia AA, Lambertucci JR, Parra JC, Katz N, Rocha RS, Gazinelli G 1986. Immune responses during human schistosomiasis. XII. Differential re- sponsiveness in patients with hepatosplenic disease. Am J Trop Med Hyg 35: 793-802.

Coura JR, Conceição MJ 1981. Correlação entre carga parasitária de $S$. mansoni e gravidade das formas clínicas em uma comunidade rural de Minas Gerais. Rev Soc Bras Med Trop 19: 69-73.

Coutinho EM 1980. Patobiologia da desnutrição nas doenças parasitárias. Mem Inst Oswaldo Cruz 75: 63-76.

Henderson GS, Nix NA, Montesano MA, Gold D, Freeman Jr GL, McCurley TL, Colley DG 1993. Two distinct pathological syndromes in male CBA/J inbred mice with chronic Schistosoma mansoni infections. Am J Pathol 142: 703-714.

Junqueira LCU, Bignolas G, Brentani R 1979. Picrosirius staining plus polarization microscopy, a specific method for collagen detection in tissue sections. Histochem J 11: 447-455.

Lichtenberg F, Sadun EH 1968. Experimental production of bilharzial pipe-stem fibrosis in the chimpanzee. Exp Parasitol 22: 264-278.

Prata A 1991. Fatores determinantes das formas anátomo-clínicas e evolução da esquistossomose, p. 3-12. In FP Castro, PRS Rocha, AS Cunha (eds), Tópicos em Gastroenterologia, MEDSI-Editora Médica e Científica Ltda, Rio de Janeiro.

Sadun EH, Lichtenberg F, Cheever AW, Erickson DG 1970. Schistosomiasis mansoni in the chimpanzee. The natural history of chronic infections after single and multiple exposures. Am J Trop Med Hyg 19: 258277.

Symmers WStC 1904. Note on a new form of liver cirrhosis due to the presence of the ova of Bilharzia haematobia. J Pathol Bacteriol 9: 237-239.

Warren KS 1966. The pathogenesis of "clay-pipe stem cirrhosis" in mice with chronic schistosomiasis mansoni, with a note on the lengevity of the schistosomes. Am J Pathol 49: 477-489. 